\title{
Evaluación diagnóstica para el análisis de programas de manejo integral de residuos sólidos urbanos en dos universidades mexicanas
}

Ruiz-Hernández, Conrado; Lupercio Lozano, Alma Delia; Bernal González, Thalía Ameyatzin Evaluación diagnóstica para el análisis de programas de manejo integral de residuos sólidos urbanos en dos universidades mexicanas

CIENCIA ergo-sum, vol. 25, núm. 3, noviembre 2018-febrero 2019| e34

Universidad Autónoma del Estado de México, México

Esta obra está bajo una Licencia Creative Commons Atribución-NoComercial-SinDerivar 4.0 Internacional.

Ruiz-Hernández, C.; Lupercio Lozano A. D. y Bernal González, T. A. (2018). Evaluación diagnóstica para el análisis de programas de manejo integral de residuos sólidos urbanos en dos universidades mexicanas. CIENCIA ergo-sum, 25(3). https://doi.org/10.30878/ces.v25n3a12 


\section{Evaluación diagnóstica para el análisis de programas de manejo integral de residuos sólidos urbanos en dos universidades mexicanas}

Diagnostic evaluation of solid urban waste programs contained in comprehensive management plans in two Mexican universities.

Conrado Ruiz-Hernández

Universidad Nacional Autónoma de México, México

cruiz@campus.iztacala.unam.mx

Recepción: 10 de marzo de 2017

Aprobación: 14 de noviembre de 2017

Alma Delia Lupercio Lozano

Universidad Nacional Autónoma de México, México

lupercioalma09@gmail.com

Thalia Ameyatzin Bernal González

Universidad Nacional Autónoma de México, México

bioamethal@gmail.com

\section{RESUMEN:}

Se realiza una evaluación diagnóstica de programas de residuos sólidos urbanos (RSU) en dos universidades mexicanas mediante un diseño cualitativo. Se contó con la participación de un responsable directo del programa de RSU de cada universidad anfitriónvisitante. Se empleó la observación y discusión en campo para evaluar cada programa con base en los rubros que conforman la cartilla. Los resultados muestran que la institución A y B obtuvieron 8 y 2 puntos respectivamente. Se concluye que, además de los aspectos técnicos y de ingeniería, es preciso el utilizar o diseñar instrumentos (cualitativos o cuantitativos) para el análisis comparativo de casos de programas incluidos en planes ambientales.

Palabras ClaVe: evaluación, programas de manejo, residuos sólidos urbanos, educación ambiental, educación superior, plan institucional.

\section{ABstract:}

Design: Qualitative. Participants and context: A direct responsibility for the RSU program of each university that participated as host-visitor. Method: Observation and discussion in the field joint evaluation of each program based on the items that make up the primer. Results: Institution A and B obtained 8 and 2 points respectively. Conclusions: In addition to the technical and engineering aspects, it is necessary to use and / or design instruments (qualitative or quantitative), for the comparative analysis of cases of programs included in plans Environmental impacts. The institutional evaluation of environmental aspects should be a constant practice in inter-institutional collaborations that favor better performance and not internal self-complacency.

KEYWORDS: evaluation, management programs, solid urban waste, environmental education, higher education, institutional plan.

\section{INTRODUCCIÓN}

México genera diariamente 102895 toneladas de residuos, de las cuales se recolecta $83.93 \%$ y se coloca en sitios de disposición final 78.54\%. Lo anterior muestra que se recicla únicamente $9.63 \%$ de los desechos.

En el país sigue predominando el manejo básico de los RSU (residuos sólidos urbanos) que consiste en recolectar y disponer los residuos en rellenos sanitarios; sin embargo, se desaprovechan aquellos que son susceptibles a reincorporarse al sistema productivo, lo que disminuiría la demanda y la explotación de nuevos recursos. En países como Suiza, Países Bajos, Alemania, Bélgica, Suecia, Austria y Dinamarca, se observa que la disposición final de los residuos en rellenos sanitarios corresponde a menos de 5\% (Semarnat, 2017). 
Dentro de las instalaciones universitarias, uno de los principales problemas es la generación de RSU, su transporte y su disposición final. Las Instituciones de Educación Superior (IES) cuentan con estructuras que las asemejan a microciudades: comercios, servicios de cafeterías, librerías, oficinas, servicios de imprenta y fotocopiado, entre otros, todo ello sumado a la actividad deportiva, cultural y académica.

En nuestro país la inserción de temáticas ambientales en los planes y en los programas de educación oficial se inició en los años noventa. Gracias a la relevancia social y al posicionamiento que han ido ganando este tipo de temáticas en la agenda política y educativa, se han creado carreras y posgrados vinculados con el medioambiente en la educación superior (Ávila, 2014).

Si bien existe un compromiso de las IES con la sustentabilidad, éste debe reflejarse en una gestión permanente que fomente la participación y crítica de la comunidad, lo que implicaría procesos de organización, negociaciones firmes y una buena disposición o apertura de los involucrados (cuestiones que son complicadas y que llevan tiempo). Para lograrlo, es necesario un enfoque integral y multidisciplinario que involucre la colaboración de diversos actores, entre los que destacan: especialistas en el tema, personas dedicadas especialmente a este cometido, académicos de diversas áreas y la comunidad estudiantil.

A pesar de contener la perspectiva ambiental en el currículo de las universidades, son escasas las IES que logran tener programas ambientales, lo suficientemente desarrollados, sobre manejo integral de RSU que $a$ ) incorporen dinámicas de planeación, desarrollo, aplicación y evaluación continua, $b$ ) involucren a los grandes sectores de su comunidad y $c$ ) cumplan con objetivos ambientales, resultados claros y vinculados con el área pedagógica. ${ }^{[1]}$

A partir del compromiso social y educativo de formar profesionales para un presente y un futuro globalizado, el alumno al egresar deberá tener las competencias para ejercer su profesión y conservar el medio ambiente.

\section{Programas ambientales en universidades}

Desde hace varios años, y de manera progresiva, se han ido desarrollando estrategias ambientales en distintas universidades, las cuales han girado en torno a la generación de residuos y a su manejo integral, pero no se han enfocado en uno de los ámbitos, también relevantes, al momento de planificar estrategias ambientales. En este sentido, se habla de la composición de los residuos. De las investigaciones y de los casos de estudio revisados, se identifica la importancia de conocer a fondo la composición de los residuos generados para el diseño y la implementación de programas de manejo que formen parte de un Plan de Manejo Integral (Smyth et al., 2010; Espinosa et al., 2008; Azuela y Preciado, 2011; Ruiz, 2012; Rosales et al., 2013).

Muestra del interés de las acciones que se han implementado al interior de las IES, en 2015 se 1 levó a cabo un encuentro académico organizado por la Universidad Autónoma Metropolitana (UAM), Unidad Azcapotzalco, y cuyo objetivo era que académicos e investigadores involucrados en el manejo de residuos en IES compartieran sus experiencias, identificaran buenas prácticas y exploraran oportunidades de colaboración.

Durante este encuentro, las universidades que presentaron sus programas de manejo de RSU fueron la Universidad Autónoma Metropolitana (UAM), en sus Unidades Azcapotzalco y Cuajimalpa, así como la Rectoría General, el Instituto Tecnológico de Toluca, la Facultad de Estudios Superiores Iztacala de la Universidad Nacional Autónoma de México (UNAM), la Universidad Veracruzana y la Universidad Autónoma de Nayarit. También, se presentaron investigaciones con temáticas diversas en el contexto de las IES tales como el manejo de residuos de jardinería, de residuos eléctricos y electrónicos, lámparas fluorescentes, la cuantificación de residuos electrónicos, así como la generación y alternativas para el manejor de los residuos de poliestireno expandido y de residuos peligrosos biológico infecciosos (Espinosa et al., 2015).

El encuentro académico dejó entrever las distintas etapas en las que se encuentran los planes o los programas de manejo de RSU, así como los principales resultados y propuestas de investigación. En este 
sentido, el manejo integral de RSU es un tema de interés actual no sólo por su importancia ambiental sino también por su carácter social, profesional o de investigación.

Como se mencionó, en nuestro país se identifican dos instituciones que se han dedicado desde hace varios años a desarrollar programas ambientales que incluyen al manejo integral de RSU dentro de sus estrategias de actuación:

a) La UAM-Azcapotzalco ha concretado algunas acciones desde octubre de 2003, entre las que destacan el Programa de Ahorro de Energía, la Protección y el Cuidado de Áreas Verdes, la Planta de Tratamiento de Aguas Residuales y el Programa de Compras "Verdes". En esas mismas fechas se puso en marcha el Programa de Manejo Integral de Residuos Sólidos Urbanos denominado "Separación, por un mejor ambiente" encaminado a concientizar a la comunidad del campus sobre la problemática de los residuos e involucrarla en su separación, así como en el cumplimiento de la Ley General para la Prevención y Gestión Integral de los Residuos (LGPGIR) y de su Reglamento. Para tales efectos, se diseñó el Plan de Manejo (PM) como un instrumento de gestión integral (GI), que contiene el conjunto de acciones y procedimientos para facilitar el acopio y la disposición de productos de consumo que al terminar su vida útil se convierten en residuos sólidos urbanos (RSU)(Espinosa et al., 2008; Espinosa et al., 2013).

b) En el caso de la UNAM, se diseñó entre 2002 y 2003 un proyecto para la caracterización y cuantificación de los residuos sólidos en Ciudad Universitaria por medio de la Unidad de Gestión Ambiental de la Facultad de Química y la Dirección General de Obras. Los objetivos principales eran, por un lado, evaluar los flujos de generación en las diferentes zonas del campus universitario (zona escolar, cultural, estadio, áreas deportivas, institutos) y, por el otro, determinar el porcentaje de residuos con potencial de aprovechamiento e identificar las alternativas más viables para que la UNAM elaborase los planes de manejo en función de la ley vigente y de la tecnología disponible (Alcántara et al., 2005).

\section{Evaluación de PROgRamas (AMbientales) EN EDUCACión SUPERIOR}

Actualmente, en la educación se hace hincapié en la evaluación institucional como parte de la calidad educativa, se evalúan programas y procesos desde la educación básica hasta la educación superior. Para esta última, existen alternativas de acreditación y certificación como una vía para generar un sistema educativo de calidad.

Según Barrera y Aguado (2007), las instituciones educativas deben propiciar una cultura de evaluación orientada a conceptualizarla como un mecanismo de mejora y de rendición de cuentas a la comunidad donde se involucran tres ámbitos:

a) La evaluación institucional: autoevaluación orientada a identificar las fortalezas y debilidades desde la perspectiva de los actores principales en el proceso educativo. Los responsables son los Comités Interinstitucionales para la Evaluación de la Educación Superior A. C. (CIEES).

b) La evaluación externa: evaluación extrainstitucional realizada por pares académicos orientada al análisis de los programas educativos o de las funciones sustantivas de la institución. Los encargados son el Consejo para la Acreditación de la Educación Superior (COPAES) u organismos internacionales.

c) La certificación de los procesos: enfocada en mejorar la eficiencia a través del cumplimiento de normas nacionales e internacionales. Dentro de las normas internacionales se encuentra el Sistema de Gestión de Calidad ISO 9001:2000 (norma internacional aceptada por organizaciones y empresas que define los requisitos mínimos que debe cumplir un sistema de gestión de calidad para ser certificado) (Barrera y Aguado, 2007). 
La evaluación es parte fundamental de una institución, de una estrategia, política, programa o proyecto, ya que brinda información relevante y da pautas para mejorar los procesos. Para el caso de una institución educativa, sería el proceso de enseñanza-aprendizaje. Para el caso de los programas de educación ambiental, las evaluaciones se pueden aplicar por dimensiones y por niveles, así como por sus fines, objetivos y resultados. Si bien existen publicaciones de estudios de programas de manejo de RSU en instituciones universitarias, no se encontraron instrumentos que permitan evaluarlos desde una visión integral. Debido a estas ausencias, se diseñó una cartilla de evaluación que considera diez rubros: $a$ ) generación, $b$ ) planeación, c) organización, $d$ ) infraestructura, $e$ ) tipología, $f$ ) instrumentación/seguimiento, $g$ ) educación/capacitación, b) evaluación, i) transparencia y j) rendición de cuentas (Ruiz et al. 2015; Ruiz y Lupercio, 2015).

En este artículo se describe la aplicación del instrumento de cartilla a partir de una evaluación externa y extrainstitucional realizada por pares de los responsables de los programas y orientada al análisis de los programas educativos o de las funciones sustantivas de la institución. Para los fines del estudio, las instituciones son denominadas Institución A (UAM-Azcapotzalco) e Institución B (UNAM-Iztacala).

La Institución A cuenta con una amplia experiencia en el manejo de RSU y estudios de generación. Desde 2003 se han desarrollado varios programas ambientales en el interior de la institución: ahorro de energía, protección y cuidado de las áreas verdes, planta de tratamiento de aguas residuales, compras "verdes" y el de separación de RSU. En 2010 ganaron el Premio Nacional al Mérito Ecológico.

Por su parte, en la Institución B se encuentra en vigor el Programa de Manejo Integral de Residuos que inició en agosto de 2014. El programa estableció una clasificación en orgánicos, inorgánicos, reciclables, PET y sanitarios. Se conformó además un comité para el análisis, discusión y la toma de decisiones. Con anterioridad ya existían iniciativas que habían logrado colocar algunos residuos en una ruta para su reutilización, como el acopio de PET y el papel de oficina (Ruiz y Lupercio, 2015).

\section{Procedimiento}

Para realizar la evaluación diagnóstica de los programas ambientales de manejo de RSU en ambas instituciones fue necesaria la integración de un equipo de evaluadores compuesto por un responsable directo de ambas instituciones que fungió como anfitrión y visitante.

La evaluación consistió en reunir al personal más relacionado con el tema de manejo de los residuos en la institución visitada (A) y con los evaluadores de la otra institución (B), donde se daban a conocer los lugares estratégicos de acopio, la clasificación en los contenedores, información escrita del programa en documentos, páginas web y, por supuesto, la experiencia demostrada en el manejo de la información. Al término se asentó $l$ a evaluación numérica de la cartilla en la que ambas partes estuvieran de acuerdo con los valores asignados en cada rubro, y se realizó la misma actividad en la universidad visitante.

\section{Resultados}

De las dos instituciones evaluadas (tabla 1) es evidente, y hasta cierto punto esperado, identificar dos niveles de avance distinto que reflejan interés, compromiso y mucho trabajo por parte de sus coordinadores.

La Institución A, que logró una puntaje alto (8), obtuvo la calificación más alta (1) en los siguientes rubros: generación, planeación, organización, infraestructura, tipología, infraestructura/seguimiento, educación/ capacitación y evaluación, lo que admite afirmar un trabajo continuo y, al parecer sistemático, ya que se han creado bases de datos confiables y consistentes que permiten interpretar el desempeño de s u comunidad y que además han logrado contar con espacios y con la infraestructura adecuada para el f uncionamiento de su programa de acopio de desechos. Los rubros que obtuvieron calificación de 0 son 1 os de transparencia $y$ rendición de cuentas, aspectos estratégicos para que un programa de manejo integral de RSU permanezca vigente y sea apoyado o legitimado por su comunidad independientemente del apoyo institucional. 
TABLA 1

Cartilla de evaluación utilizada para evaluar de la Institución A y la Institución B

\begin{tabular}{|c|c|c|c|}
\hline \multirow[t]{2}{*}{ Punto evaluado } & \multirow{2}{*}{$\begin{array}{c}\text { Descripción breve de lo que se evalúa } \\
\text { (el equipo evaluador puede ampliar los detalles a } \\
\text { observar en cada punto) }\end{array}$} & \multicolumn{2}{|c|}{ Calificación } \\
\hline & & $\mathrm{A}$ & B \\
\hline a) Generación & $\begin{array}{l}\text { Determinación de desechos generados y estimación } \\
\text { de cantidades aproximadas. Conviene dar a conocer el } \\
\text { método aplicado. }\end{array}$ & 1 & 0 \\
\hline b) Planeación & $\begin{array}{l}\text { Diseño de un plan de manejo integral de la basura } \\
\text { (reducción, reúso y reciclaje) con metas a corto, } \\
\text { mediano y largo plazo que sea factible de ser adoptado } \\
\text { por la comunidad. Este plan debe ser del dominio } \\
\text { público. Conviene que para este cometido se } \\
\text { establezcan convenios con instancias de gobierno y } \\
\text { empresas que encaucen los desechos recaudados y } \\
\text { sean aprovechados de manera óptima. }\end{array}$ & 1 & 0.5 \\
\hline c) Organización & $\begin{array}{l}\text { Decidir sobre la manera en que el programa se pondrá } \\
\text { en marcha en la institución y al personal que se } \\
\text { encargará de su aplicación. }\end{array}$ & 1 & 0.5 \\
\hline d) Infraestructura & $\begin{array}{l}\text { Nombramiento de personal honorario o contratado, } \\
\text { así como disponer de los recursos materiales } \\
\text { necesarios que cuiden la salvaguarda de los } \\
\text { participantes. }\end{array}$ & 1 & 0.5 \\
\hline e) Tipología & $\begin{array}{l}\text { Manera en que se pide a la comunidad el vertido de la } \\
\text { basura, en donde haya claridad y entendimiento sobre } \\
\text { lo que deben hacer las personas. Conviene dar a } \\
\text { conocer cómo se tomaron decisiones. }\end{array}$ & 1 & 0.5 \\
\hline $\begin{array}{l}\text { f) Instrumentación/ } \\
\text { seguimiento }\end{array}$ & $\begin{array}{l}\text { Aplicación del plan de manejo diseñado y supervisión } \\
\text { sobre su marcha apropiada. }\end{array}$ & 1 & 0 \\
\hline $\begin{array}{l}\text { g) Educación/ } \\
\text { capacitación }\end{array}$ & $\begin{array}{l}\text { Intervenciones educativas pedagógicamente bien } \\
\text { diseñadas respecto al aprendizaje de conocimientos y } \\
\text { habilidades vinculadas a la cultura del reciclaje. }\end{array}$ & 1 & 0 \\
\hline h) Evaluación & $\begin{array}{l}\text { Medición con la mayor objetividad posible del } \\
\text { aprendizaje y de la motivación que manifiesta la } \\
\text { comunidad. Esta información debe ser útil para } \\
\text { retroalimentar el programa. }\end{array}$ & 1 & 0 \\
\hline i) Transparencia & $\begin{array}{l}\text { Mecanismos que dan información amplia a la } \\
\text { comunidad sobre la marcha del programa y de } \\
\text { problemas que es necesario resolver. }\end{array}$ & 0 & 0 \\
\hline $\begin{array}{l}\text { j) Rendición de } \\
\text { cuentas }\end{array}$ & $\begin{array}{l}\text { Información fidedigna sobre los recursos económicos } \\
\text { o en especie obtenidos, a través del programa, y el } \\
\text { destino que se les da. }\end{array}$ & 0 & 0 \\
\hline
\end{tabular}

Fuente: elaboración propia.

Nota: se evalúa cada aspecto con 1 punto cuando el rubro es claramente atendido en la institución, se asigna 0.5 cuando se atiende de manera incompleta, pero con avances notorios, y 0 cuando el detalle es sólo incipiente o desatendido. 
Para la Institución B los puntajes en los cuales se otorgó una calificación de medio punto (0.5) fueron en planeación, organización, infraestructura y tipología, que en conjunto sumaron 2. La calificación es baja debido a que no se han logrado atender de manera óptima estos rubros. Respecto a esta institución, se identificó que algunas de las iniciativas sobre el manejo de algunos residuos en la actualidad se llevan a cabo principalmente por trabajadores y jefes de servicio que van en línea con el Programa de Manejo Integral de Residuos y con 1 os esfuerzos, quienes son impulsados por la administración actual para el mejoramiento de las instalaciones de la escuela.

Asimismo, se localizaron estudios de generación de carácter escolar que, gracias al esfuerzo e interés de profesores y alumnos, se han concretado. Sin embargo, dentro del programa no se ha concretado una sistematización continua de datos a partir de la generación y el manejo de los RSU, lo que impide establecer metas realistas a corto y mediano plazo, pues hace falta mayor información sobre el desempeño de la comunidad que asiste con regularidad a esta universidad. Estas falencias se deben, en parte, a que el programa de manejo de RSU de la institución se encuentra en proceso de gestación y diseño.

El diseño y aplicación de la cartilla implementada en este estudio incorpora aspectos socioeducativos y de participación comunitaria ausentes normalmente en los reportes técnicos sobre el manejo de los residuos sólidos urbanos. En los reportes técnicos localizados no se incluyen de manera clara detalles como los aprendizajes promovidos en la comunidad, transparencia y rendición de cuentas, entre otros aspectos importantes que sí se incluyen en la cartilla aplicada. El procedimiento de evaluación cooperativa, que en este reporte se expone, subsana la ausencia de datos cualitativos en este tipo de abordajes y se constituye así en un aporte original tanto nacional como internacionalmente.

\section{ConCLUSIONES}

El diseño, aplicación y mejoramiento de este tipo de instrumentos de evaluación, como la cartilla utilizada puede ofrecer una perspectiva que permita hacer un alto y reconocer lo que se hace bien y qué se puede mejorar de manera muy puntual, pues es un abordaje original de un tipo de evaluación comparada y coparticipativa en programas de manejo de RSU.

Se hace evidente que los programas evaluados se encuentran en momentos distintos de diseño y aplicación y, por ende, la experiencia de cada una no es homogénea. Esta situación se expresa en la calificación que obtuvieron.

Considerar la evaluación institucional, que incluya y desarrolle aspectos como la acreditación de programas en el manejo de RSU en instituciones de educación superior, sería un gran avance para la educación ambiental, ya que los procesos de evaluación externa y de certificación en universidades posibilitarían el crecimiento y la mejora del programa ambiental, así como el equilibrio de los desbalances que existen en la evaluación i nterna caracterizada por sus límites en cuanto a sus formas (contenido) e instrumentos que no permiten una autocrítica. Por lo tanto, la perspectiva unilateral que la propia institución tenga de su tarea ambiental es parcial, situación que limita el alcance de su programa.

Este tipo de ejercicios de investigación como el llevado a cabo facilita el análisis crítico de los resultados para la toma de decisiones a corto, mediano y largo plazo, ya que permite al mismo tiempo un trabajo conjunto entre instituciones, lo cual favorece el conocimiento y el reconocimiento de experiencias, acciones y procesos en la toma de decisiones en la aplicación, en el desarrollo y en la estructura del programa ambiental.

La Institución A cuenta con un Plan Institucional Ambiental que rige y da sentido al programa de manejo de residuos debido a que se sustenta en las bases teóricas y epistemológicas que se han desarrollado 
expresamente por la institución, lo que permite tener un sentido claro de a dónde llegar, por qué y, sobre todo, los fines ambientales que se pretenden lograr.

La Institución B, si bien cuenta con un incipiente programa, aún no ha desarrollado un Plan Institucional Ambiental que sustente y oriente las acciones ambientales, lo que puede retardar las metas y los objetivos destinados a la conservación del medioambiente en la institución. Por lo tanto, la creación y el desarrollo de un plan institucional favorecerían la orientación del programa de recuperación de residuos, abriría la posibilidad de abordar otras áreas y la elaboración de programas complementarios de ahorro de luz, agua, etcétera. De igual manera, fortalecería los vínculos entre los docentes interesados en este ámbito y los estudiantes para desarrollar investigaciones o propuestas innovadoras e interdisciplinarias para el manejo de RSU.

La orientación que se está dando en la educación a partir de la última reforma educativa es incluir la evaluación no sólo a los procesos educativos, en los cuales es indispensable, sino también en la labor docente y los programas formativos. Si bien existe polémica al respecto, consideramos que en el ámbito de la educación ambiental es indispensable integrar la evaluación en las diferentes fases de los programas, en este caso de manejo de RSU, pero no sólo interna sino también en colaboración con una o más instituciones con los mismos intereses de conservación y cuidado del medioambiente.

Los resultados que ofrece la cartilla utilizada aportan indicadores del estado del programa en el momento de la evaluación, así como la posibilidad de discusión y negociación con los evaluadores, quienes también conocen del tema y tiene un programa en su universidad. Por lo tanto, es una base útil que puede desarrollarse con mayor detalle por los participantes de la evaluación, puesto que el instrumento posibilita la flexibilidad en la identificación de áreas que se deben mejorar y, en su caso, favorece el reconocimiento de aquellos aspectos que han sido desarrollados de manera óptima; suele denominarse como la repetición de las "buenas prácticas".

Por lo tanto, este instrumento es un aporte original que permite, por un lado, el desarrollo de instrumentos más elaborados y, por el otro, considerar la evaluación institucional como un proceso continuo que ofrece la posibilidad de hacer diagnósticos realistas del estado en que se encuentran los programas ambientales de residuos sólidos urbanos.

\section{ANÁLISIS PROSPECTIVO}

La cartilla de RSU descrita y aplicada en dos instituciones educativas de nivel superior es un prototipo de instrumento útil para evaluar y mejorar los programas institucionales para el manejo integral de los RSU inserto en un programa de educación ambiental que va más allá de las pretensiones de los burocráticos programas municipales.

Las instituciones de educación superior en nuestras sociedades, tanto en una perspectiva nacional como internacional, deben constituirse en modelos a seguir en el manejo verdaderamente eficaz y con perspectiva social de los RSU, dado que en ellas se reúnen un cúmulo de saberes científicos, tecnológicos y humanísticos que son necesarios para afrontar a este tipo de problemas. La cartilla, así aplicada y con participación crítica, puede ser un elemento coadyuvante para el logro de este cometido. Verdaderamente, la unión hace la fuerza.

\section{Agradecimientos}

Los autores hacemos un reconocimiento a las facilidades otorgadas para la elaboración de este artículo a la Universidad Autónoma Metropolitana, Unidad Azcapotzalco, y en especial al Ingeniero Roberto Carlos Vázquez Solís. También agradecemos a la Facultad de Estudios Superiores Iztacala por el apoyo brindado, y en particular a la Contadora Ferrer, al Arquitecto Chirinos, al Maestro Carlos Palacios y al Doctor Francisco José Torner. 


\section{ReFERENCIAS}

Alcántara, V., Cruz, I. y Santos, E. (2005). Caracterización y cuantificación de los residuos sólidos en Ciudad Universitaria. Congreso Interamericano de Residuos. Mérida, Yucatán. 4-7 de mayo.

Ávila, L. (2014). Los programas ambientales universitarios en México. Entre el discurso ambiental y negocios verdes. Sociedad y Ambiente, 1(3), 26-52.

Azuela, J. y Preciado, S. (2011). Diagnóstico de generación de residuos sólidos urbanos y estimación beneficios económicos y ambientales de la implementación de un sistema de reciclaje en la universidad de Monterrey (UDEM). Centro de sustentabilidad UDEM.

Barrera, M. y Aguado, G. (2007). La evaluación integral de programas y procesos: un camino hacia la calidad, Educere, 11(37), 209-215.

Espinosa, V. R. M., Turpin, M. S., Polanco, S. G., de la Torre, V. A., Delfín, A. I. y Raygoza, M. I. (2008). Integral urban solid waste management program in a mexican university. Waste Manage, 28, 27-32.

Espinosa, V. R., Turpin, M. S., Vázquez, S. R., Vázquez, M. A., Cisneros, R. A., de la Torre, V. A., García, G. B. (2013). La gestión ambiental en una institución de educación superior asociada a las prácticas de separación y recuperación de residuos. Revista Internacional de Contaminación Ambiental, 29(3) 49-57.

Espinosa, V. R., Valdemar, S. y Vázquez, A. (Comps.) (2015). Encuentro de expertos en residuos sólidos. Encuentro académico sobre manejo de residuos en universidades. Sociedad Mexicana de Ciencia y Tecnología Aplicada a residuos sólidos, A. C. Disponible en http://www.somers.ac.org.

Rosales, M., Saldaña, C., Toledo, V. y Maldonado, L. (2013). Caracterización y potencial del reciclado de los residuos sólidos urbanos generados en el Instituto Tecnológico de Tepic, una institución de educación superior. Revista Bio Ciencias, 2(3), 216-123.

Ruiz, C., Torner, F., Lupercio, A y T. Bernal (2015). Auditoría ambiental participativa. Cartilla de RSU, Ciencia y Desarrollo, 41(275), 34-35

Ruiz, C. y A. Lupercio (2015). Auditoría ambiental participativa sobre manejo de RSU en dos universidades mexicanas: Prospección preliminar. Memoria del primer encuentro académico sobre manejo de residuos en universidades. México: UAM Azcapotzalco.

Ruiz, M. (2012). Caracterización de residuos sólidos en la universidad Iberoamericana, Ciudad de México. Revista Internacional de Contaminación Ambiental, 28(1), 93-97.

Smyth, P. D., Fredeen, A. L., Booth, A. L. (2010). Reducing solid waste in higher education: The first step towards 'greening' a university campus. Resources, Conservation and Recycling, 54, 1007-1016.

Semarnat (Secretaría del Medio Ambiente y Recursos Naturales) (2017). Residuos Sólidos Urbanos y manejo especial. Consultado el 28 de junio de 2017. Disponible en http://www.gob.mx/semarnat/acciones-y-programas/residu os-solidos-urbanos-rs

\section{Notas}

[1] Desde el 2000 se constituyó el Consorcio Mexicano de Programas Universitarios para el Desarrollo Sustentable (Complexus). Actualmente está conformado por 18 universidades a lo largo de la República Mexicana. Disponible en http://www.complexus.org.mx/

\section{BY-NC-ND}

